Vol. 4, Special Issue 2, January 2017

\title{
Full Automatic Threading Machine with AVR Control
}

\author{
Apshinge Namrata ${ }^{1}$, Joshi S.S. ${ }^{2}$ \\ Student E\&TC Department, Dr. Daulatrao Aher College of Engineering, Karad, India ${ }^{1}$ \\ Assistant Prof, E\&TC Department, Dr. Daulatrao Aher College of Engineering, Karad, India ${ }^{2}$
}

\begin{abstract}
The vibration of threading machine is major problem in Kalburgi Industry, to overcome the problem by our project. So we have to design this system," Threading machine automated with AVR control". The manually operating threading machine is automated with the help of pneumatic actuator. The AVR used as control system. The objects are clamped and declamped using pneumatic actuator. In a pneumatic system the working fluid is a gas (mostly air) which is compressed above atmospheric pressure to impart pressure energy to the molecules. This stored pressure potential is converted to a suitable mechanical work in an appropriate controlled sequence using control valves and actuators. Due to this reduce vibration problem which is main object of our project.
\end{abstract}

Keywords: Pneumatic actuator, control system, automatic threading.

\section{INTRODUCTION}

The $21^{\text {st }}$ century is said to be century of inventions, automation. Now, a days automation plays important role in industry. Automatic control system, which saves manpower and financial statement \& provide better accuracy. Hence we have selected the project "Full Automatic Threading Machine with AVR control."

The threading system used for automation is installed in Mechanical workshop of Institution. The system includes actuating elements like pneumatic actuator, direction control valve, proximity sensor, and it is programmed effectively using AVR. The manually operating threading machine is automated with the help of pneumatic actuator. There is clamping and declamping of machine to thread the object by using actuator. When thread the object, there is major vibration problem. The vibration of threading machine into up and down motion with full axis.

There is a need to develop automatic control system for threading machine. These done with PLC or Raspberry Pi also. But PLC (Programmable Logic Controller) becomes more expensive project. With Raspberry Pi also increases more complexity for project. It is like mini computer. Using Raspberry Pi, reboot the system every time and these not possible to reboot every time. So we have to design system with AVR.

\section{PROPOSED SYSTEM}

\section{a. Block Diagram Description}

$12 \mathrm{~V}$ 10A SMPS power supply is required for project. Proximity Sensors is a sensor able to detect presence of nearby objects without any physical contact. Proximity sensors emit electromagnetic field or beam of electromagnetic radiation and looks for changes in field or return signal. The object being sensed is referred to proximity sensors target.

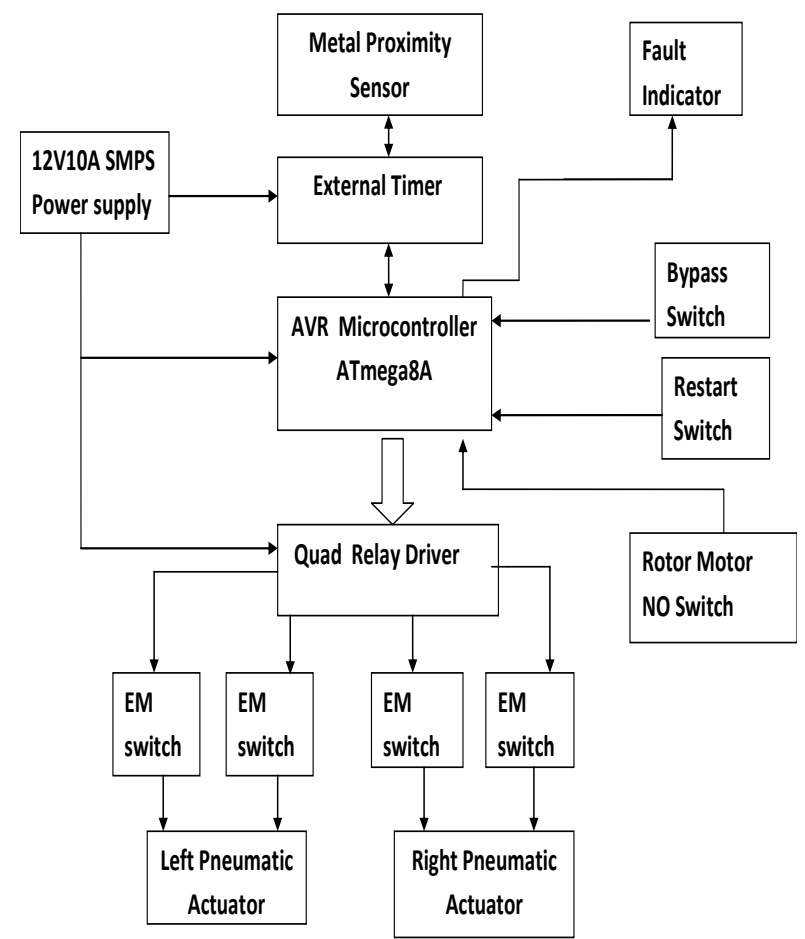

Fig.1.Proposed system

Proximity sensor can have high reliability and long functional life because of absence of mechanical parts and lack of physical contacts between sensed objects. They used in machine vibrations monitoring to measure the variation in distance between a shaft and its support bearing.

AVR(Automatic Voltage Regulator)is a hardware device used to maintain voltage to electronic device. Atmel AVR core combines instruction set with 32 general purpose working register. These directly connected to ALU allow 
him to independent registers to be accessed in one single hydraulic. In a pneumatic system the working fluid is a gas inst. executed in one clock cycle.AVR uses Harvard (mostly air) which is compressed above atmospheric architecture with separate memories and buses for pressure to impart pressure energy to the molecules. This programming data. stored pressure potential is converted to a suitable Quad Relay driver used to increases current limit. Then mechanical work in an appropriate controlled sequence rotor motor $\mathrm{NO}$ (normally open) switch is connected to using control valves and actuators. Pneumatic systems are starter of threading machine motor. Due to this made well suited for the automation of a simple repetitive task. normally closed connection. By using bypass switch, if The working fluid is abundant in nature and hence the circuit not active then also machine run successfully. In running and maintenance cost of these systems are this project, pneumatic actuator used to reduce the exceptionally low. All fluids have the ability to translate vibration of threading machine into an up and down and transfigure and hence pneumatic systems permit motion platform to form a reconfigurable full -axis. variety of power conversion with minimal mechanical Pneumatic systems form the most primitive and distinct hardware. Conversion of various combinations of motions class of mechanical control engineering. They are like rotary-rotary, linear-rotary and linear-linear is classified under the term 'Fluid Power Control', which possible[4]. The simplicity in design, durability and describes any process or device that converts, transmits, compact size of pneumatic systems make them well suited distributes or controls power through the use of for mobile applications. These features make them pressurized gas or liquid. When high-pressure liquids (like versatile and find universal applications. oil) are used to transmit power, the system is termed as

\section{FLOWCHART}

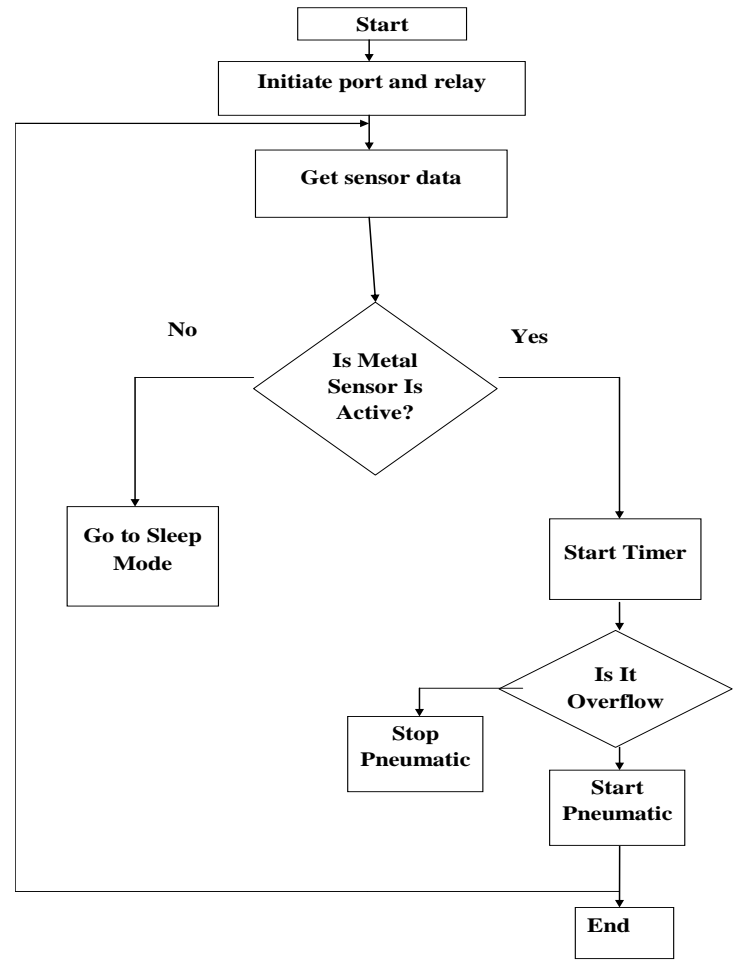

\section{FLOW CHART DESCRIPTION}

When supply pass to thread machine, initiate ports \& relays into the circuitry. When object is placed into the machine to thread it. These object is sensored by proximity sensor without any physical contact. If metal sensor actives then starts timer to thread the object. Otherwise system stop its operation. Then pneumatic actuator starts work, where air compressed above atmospheric pressure. Then stored pressure converted into suitable mechanical work. The process starts continuously and thread the object one by one.

\section{HARDWARE REQUIREMENTS}

AVR Microcontroller-ATmega8A

The Atmel ATmega8A is a low-power CMOS 8-bit microcontroller based on the AVR enhanced RISC architecture. By executing powerful instructions in a single clock cycle, the ATmega8A achieves throughputs close to 1MIPS per MHZ. This empowers system designer to optimize the device for power consumption versus processing speed. The Atmel AVR core combines a rich instruction set with 32 general purpose working registers. 
All the 32 registers are directly connected to the There is no electrical connection inside the relay between Arithmetic Logic Unit (ALU), allowing two independent the two circuits; the link is magnetic and mechanical. The registers to be accessed in one single instruction executed coil of a relay passes a relatively large current, typically in one clock cycle. The resulting architecture is more code $30 \mathrm{~mA}$ for a $12 \mathrm{~V}$ relay, but it can be as much as $100 \mathrm{~mA}$ for efficient while achieving throughputs up to ten times faster than conventional CISC microcontrollers.

The ATmega8A provides the following features: $8 \mathrm{~K}$ bytes of In-System Programmable Flash with Read- WhileWrite capabilities, 512 bytes of EEPROM, $1 \mathrm{~K}$ byte of SRAM, 23 general purpose I/O lines, 32 general purpose working registers, three flexible Timer/Counters with compare modes, internal and external interrupts, a serial programmable USART, one byte oriented Two-wire Serial Interface, a 6- channel ADC (eight channels in TQFP and QFN/MLF packages) with 10-bit accuracy, a programmable Watchdog Timer with Internal Oscillator, an SPI serial port, and five software selectable power saving modes.

\section{Metal Proximity Sensor}

Proximity sensor can have high reliability and long functional life because of absence of mechanical parts and lack of physical contacts between sensed objects. They used in machine vibrations monitoring to measure the variation in distance between a shaft and its support bearing.

\section{IC 555 Timer}

IC 555 is very commonly used IC for generating accurate timing pulses. It is an 8 pin timer IC and has mainly 3modes of operation: monostable, astable, bistable. In a monostable mode time delay of pulses can be precisely controlled by an external resistor and capacitor where as in astable mode frequency and duty cycle are controlled by two external resistors and capacitors. IC 555 commonly used for generating time delays and pulses.

IC 555 with the pins as shown in the diagram. Relays allow one circuit to switch a second circuit, which can be completely separate from the first. For example a low voltage battery circuit can use a relay to switch a $230 \mathrm{~V} \mathrm{AC}$ mains circuit.

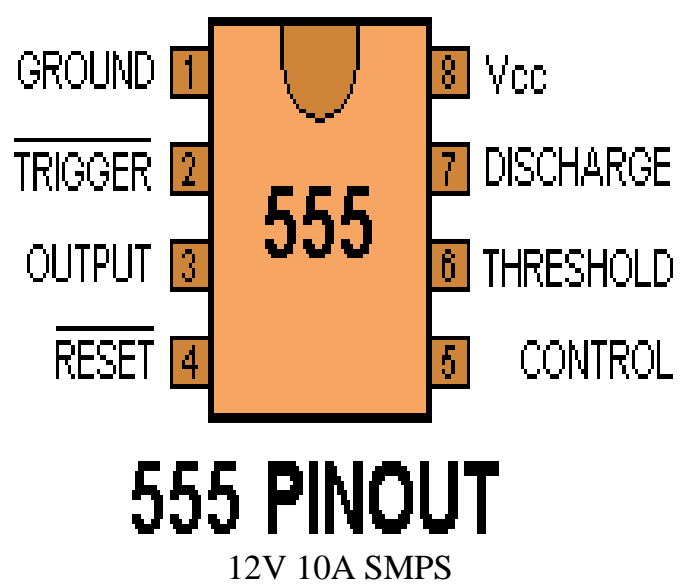
relays designed to operate from lower voltages. Most ICs (chips) cannot provide this current and a transistor is usually used to amplify the small IC current to the larger value required for the relay coil. The maximum output current for the popular 555 timer IC is $200 \mathrm{~mA}$.

\section{ULN2003 Relay Driver}

A relay is an electromagnetic switch operated by a relatively small electric current that can turn on or off a much larger electric current. The heart of a relay is an electromagnet (a coil of wire that becomes a temporary magnet when electricity flows) Output of micro-controller is not sufficient to drive the relay directly. Therefore to drive the relay we are using relay driver block by using transistor as switch. A relay is an electrically operated switch.

Current flowing through the coil of the relay creates a magnetic field, which attracts a lever and changes the switch contacts. The coil current can be on or off so relays has two switch positions and they are double throw (changeover) switches. The two spring contacts on the left and the electromagnet coil on the right. In this relay, when a current flows through the coil, it turns it into an electromagnet. The magnet pushes a switch to the left, forcing the spring contacts together, and completing the circuit they're attached to.

A relay is an electromagnetic switch operated by a relatively small electric current that can turn on or off a much larger electric current. The heart of a relay is an electromagnet (a coil of wire that becomes a temporary magnet when electricity flows) Output of micro-controller is not sufficient to drive the relay dir Electromagnetic Pneumatic Switch

\section{IV.SOFTWARE REQUIREMENTS}

\section{VP812 Universal Burner}

AVR Programmer

The programmer should work with all AVRs that can be programmed with AVR ISP interface. The programmer is powered by $5 \mathrm{~V}$ USB power bus, and it is intended for programming AVRs that are running at close to $5 \mathrm{~V}$. VP812 is an excellent USB universal programmer for AVR ICs.

\section{Express PCB}

PCB is a free and open source software suite for electronic design automation - for printed circuit boards layout. PCB was first written by Thomas Nau for an Atari ST in 1990. Initially PCB was not intended to be a professional layout system but as a tool for individuals to do small scale development of hardware. 


\section{CONCLUSION}

In this paper, pneumatic actuator used to reduce the vibration of threading machine. We successfully determined the effects of the drive shafts of the platform on the platform attitude. Then, the platform attitudes were measured. Scope of the present work is about using AVR in threading machine leads to Optimization process and threading is smooth and less noise when compared to other machine. The operator feels more combatable with AVR controller.

\section{ACKNOWLEDGMENT}

We would sincerely thank to our guide Miss S.S. Joshi for her valuable guidance, constant assistance, support endurance and constructive suggestions for the betterment of this platform of research. We also wish to thank all the staff members of the department of E\&TC engineering for helping us directly or indirectly in completing this work successfully.

\section{REFERENCES}

1) Annapurna Publishers,pp.120-228, Dey, A. (1995), "threading machine", Latest development of heavy earth moving machinery.

2) P. Chevtsov, S. Higgins, S. Schaffer and D. Seidman, "PLC Support Software at Jefferson Lab," Jefferson Lab.

3) P. A. Mehta, V. S. Samse, Dr. A. D. Rahulkar" Automatic Threading System", International Journal for Technological Research in Engineering, March 2014.

4) Naresh Raghavan, "Pneumatic Control For Robotics And Industrial Automation." 